\title{
A Printed Circuit Board Gradiometer Current Transformer
}

\author{
A Salisbury ${ }^{1}$, A J Peyton ${ }^{1}$, A Dytch $^{2}$ \\ ${ }^{1}$ Engineering department. Lancaster University, Lancaster, LA1 4YR, UK. \\ E-mail : a.salisbury@lancaster.ac.uk \\ ${ }^{2}$ Landis+Gyr Limited. \\ Hortonwood 30. Telford. Shropshire. TF1 7YG. \\ E-mail : tony.dytch@siemens.co.uk
}

\begin{abstract}
This paper describes a gradiometer type sensor for current measurement that can be realized on a printed circuit board (PCB). Key aspects of the sensor are covered such as shielding from external sources, sensitivity mapping to determine the spatial response of the system, harmonic waveform testing, and alternative designs using multiple PCB layers.
\end{abstract}

\section{INTRODUCTION}

The measurement of current is fundamental to electrical engineering and there is a mature market for $\mathrm{kW}$-hour energy meters, each including a current sensor, with approximately 700 million new units supplied per annum worldwide. The domestic market in the UK is very price conscious and demands low cost units that can meet defined standards for safety and performance [1]. This work describes an inductive based current measuring device [2] that can be realized with conventional printed circuit board (PCB) techniques. The device is contact-less and has the cost advantage of avoiding a separate current sensing element, such as a Hall device, Current transformer, Rogowski coil, Optical current technique or a Current shunt. A key design issue with this new device is to avoid interference from magnetic fields created by external currents. Recently, a PCB based device has been described $[3,4]$, which is based on a Rogowski coil and therefore encircles the current carrying conductor. The device described herein is a planar coil configuration type sensor, that with simple shielding to avoid external interference or fraudulent intentions, can be realized at low cost for mass domestic or industrial metering applications.

\section{PRINCIPLE}

The PCB sensor, figure 1 , is designed for currents $(\approx 100$ A) and low frequencies $(\approx 10 \mathrm{~Hz}$ to $1 \mathrm{kHz})$, such as is useful in household metering applications. The device is inductive and therefore has no D.C response. The lowest frequency of operation is determined by either $1 / \mathrm{f}$ noise or the integration time constant of the signal conditioning circuitry. The maximum operating frequency is dependant on selfresonance of the detector coils, and the frequency response of the conditioning circuitry may easily be several $\mathrm{MHz}$.

The sensors coils are based on a figure of eight configuration [2]. The sensor detects the $\mathrm{AC}$ current by direct inductive pick-up, exploiting Faraday's law, (i.e)

$$
e=-n \frac{d \phi}{d t}
$$

where $e$ is the induced e.m.f, $\mathrm{n}$ is the number of turns and $\phi$ is the magnetic flux linkage.

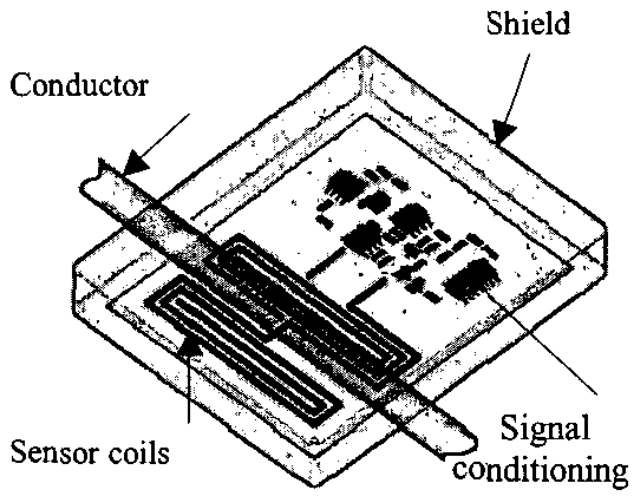

Fig.1. Diagram of the printed circuit board inductive sense loops and conditioning circuitry.

The gradiometer type configuration ensures that the induced signal for the flux round the conductor in each half of the sensor are additive. The main disadvantage with this method is that the sensor may be prone to external interference from nearby current sources, by virtue of the gradient of the field they produce. This is because the device does not measure the complete line integral of the magnetic field strength $\mathrm{H}$ over a closed loop around the conductor, (i.e)

$$
\oint H . d l
$$

unlike established inductive techniques.

Although the sensor is prone to external interference, this is significantly reduced by the coil configuration and by the use of a suitable magnetic shield. The shield should be made of a material of as high permeability as possible within the limits of its magnetic saturation, by the fields it is intended to shield. However, the possibility of magnetic saturation of the shield is remote owing to its high shape-demagnetizing factor, resulting in a lower effective permeability. The shield encloses the flux path from the wanted current source (internal current carrying conductor), and provides a low reluctance path for the magnetic field from the external current sources to flow around the shield, and hence away 
from the sensor. Figure 2 shows a spatial sensitivity response of the device with respect to the position of the current carrying conductor, with the plots scaled to $100 \%$ of this value representing the maximum sensor output at the symmetrical central position.

These plots were obtained by simulation using a model based on the Biot-Savart Law. Similar results have been obtained by practical measurements and by $3 \mathrm{D}$ finite element analysis simulations. For the one turn device the sensors output is very small $(\mu \mathrm{V})$, hence naturally a larger signal can be obtained by adding extra turns or by increasing the dimensions of the coils. In addition, experiments have shown that by varying the number of turns of the coils, the output voltage is increased in a linear fashion.

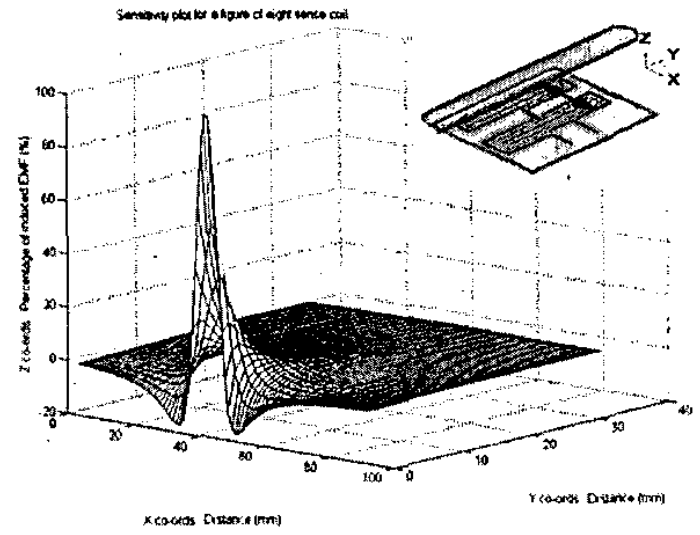

Fig.2. Sensitivity spatial response of a single layer design.

By observing figure 2 , it is evident that the sensor must be positioned accurately within $(1 \mathrm{~mm})$, in order to obtain maximum magnitude in the symmetrical position, if utilizing the uniformly standard wound coils. However, investigations have indicated that a: substantially flat sensitivity response can be obtained of over several mm's at the central position shown in figure 3 , by optimizing the turns distribution of the coils, note the turns in both coils are biased towards the inner section.

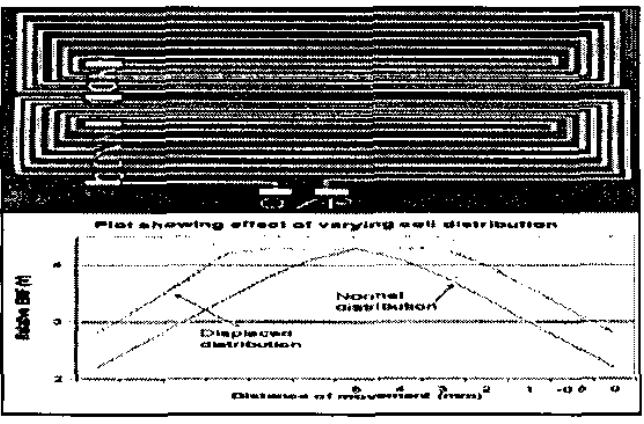

Fig.3. Plot showing distribution variation of the coils.
The calibration required depends on the configuration of the sensors, (i.e) single/twin/multi layer designs. Generally a known current is allowed to flow and then measured by the sense coils, the signal is then integrated to reverse the differentiated effect via faradays law, and then compared in magnitude to the known current, the difference found is the calibration scaling factor. Due to the linearity within the system the scaling factor value is identical for all values of current.

\section{SHIELD DESIGN}

A key design issue with the sensor is the rejection of external interference, such as that from a nearby current source. This section demonstrates the use of shields that reduce the external interference $<1 \%$ full scale, which is required for domestic metering applications. The possibility of an external interference denotes that some form of magnetic shielding is required. It is assumed that any external interference provides a magnetic field, therefore a magnetic material is necessary to prevent this erroneous field coupling with the internal sensor coils. The coils are very sensitive to magnetic fields in the parallel plane, and less sensitive in the vertical plane, therefore to provide a low tolerance to external fields, the sensor coils require as much shielding encapsulation as possible.

(a)
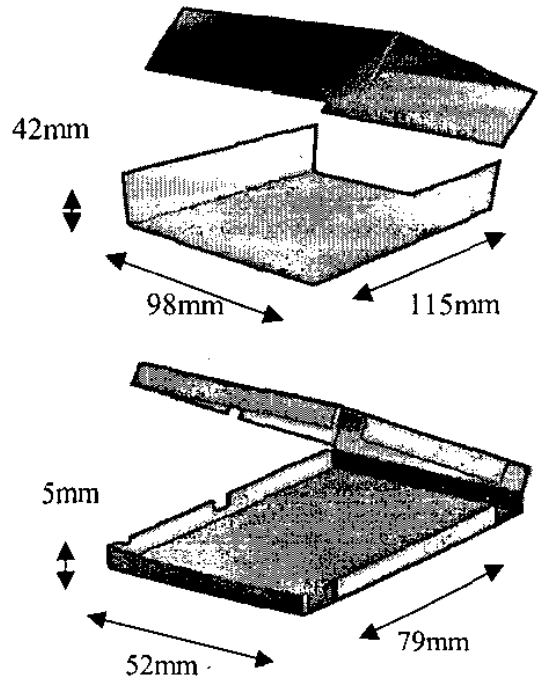

Fig.4. (a) External C-shape shield (b) Internal shield.

Hence the shield designs are such, that the magnetic fields are persuaded to flow around the shield and away from the coils. A variety of shield designs and materials were tested, with two of these designs shown in figure 4 . The optimum two materials established utilizing these designs were grainoriented silicon steel (grade 1), and standard mild steel (grade EN3A). In one form an external C-shape shield encloses the 
entire meter, where as the internal shield only encloses the internal sensor with $5 \mathrm{~mm}$ separation.

Both materials were individually tested with each shield design, and provided similar results, although, the internal shield provided the optimum results and obtained the required $<1 \%$ tolerance to external fields utilizing either material. The sizes of the shields are not fixed, and can be altered to fit virtually any system. The dimensions given are for standard class A current meters, and an overall sensor of approximately $77 \times 50 \mathrm{~mm}$.

Figure 5 shows the effect of the external shield in reducing interference from external currents, in this case a commercial silicon steel was used. The graph shows three plots i) Without shield, ii) $1 \mathrm{~mm}$ Mild steel shield, iii) $1 \mathrm{~mm}$ thick silicon steel $(\mu \mathrm{r}=8000)$, surrounding the outer case, having dimensions $38 \times 95 \times 112 \mathrm{~mm}$ of the device. The shielding reduces the interference to $0.75 \%$ of full-scale range for the worst-case condition, when the external conductor carrying 100 amps is pressed against the case of the device, and is as close as physically possible to the sense coils, and parallel to the symmetrical position of the coils in the most sensitive region.

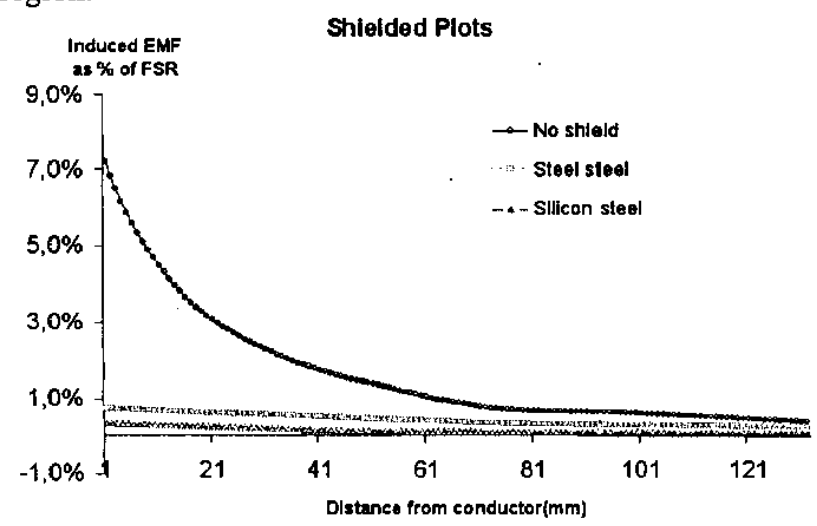

Fig.5. Sensitivity roll off to various shields.

Table 1 indicates relevant performance utilizing both material types mentioned in the internal configuration, on the single layer design. The coil sizes used were $72 \times 19 \mathrm{~mm}$ per coil with an amplification of 50 .

Table I (Sensor output from both magnetic shields, with 100 amps in interfering external conductor).

\begin{tabular}{|c|c|c|c|}
\hline $\begin{array}{c}\text { Single } \\
\text { layer design }\end{array}$ & $\begin{array}{c}\text { Internal } \\
(1.6 \mathrm{~mm})\end{array}$ & $\begin{array}{c}\text { External } \\
(20 \mathrm{~mm})\end{array}$ & $\begin{array}{c}\text { Percentage } \\
\text { difference }(\%)\end{array}$ \\
\hline No shield & 1.12 Vpk-pk & 0.126 Vpk-pk & $11.25 \%$ \\
\hline $\begin{array}{c}\text { Mild steel } \\
\text { shield }\end{array}$ & 2.6 V pk-pk & 0.020 Vpk-pk & $0.75 \%$ \\
\hline $\begin{array}{c}\text { Silicon steel } \\
\text { shield }\end{array}$ & 2.65 Vpk-pk & 0.022 Vpk-pk & $0.84 \%$ \\
\hline
\end{tabular}

It can be seen how the internal voltage output increases as the shield is incorporated, and equally, the external interference decreasing by a considerable amount. Figure 6 shows a $3 \mathrm{D}$ simulation sensitivity plot of the shielded and unshielded cases for the internal shield. The unshielded case confirms the sensitivity response to fields external to the sensor, providing evidence for the practical results and indicating the apparent need of a shield if required. The grain oriented silicon steel and standard mild steel both provide almost virtually identical results, both providing a reduction in external interference to less than $1 \%$ tolerance of full scale range.

\section{TWIN LAYER DISTRIBUTION}

The previous sections have described the sensor using a single layer PCB design approach. This section introduces the use of multiple PCB layers. In the first example a twin layer design will be investigated, one layer will be placed $4 \mathrm{~mm}$ above the internal conductor, and the other layer $4 \mathrm{~mm}$ below the internal conductor shown in figure 7 .

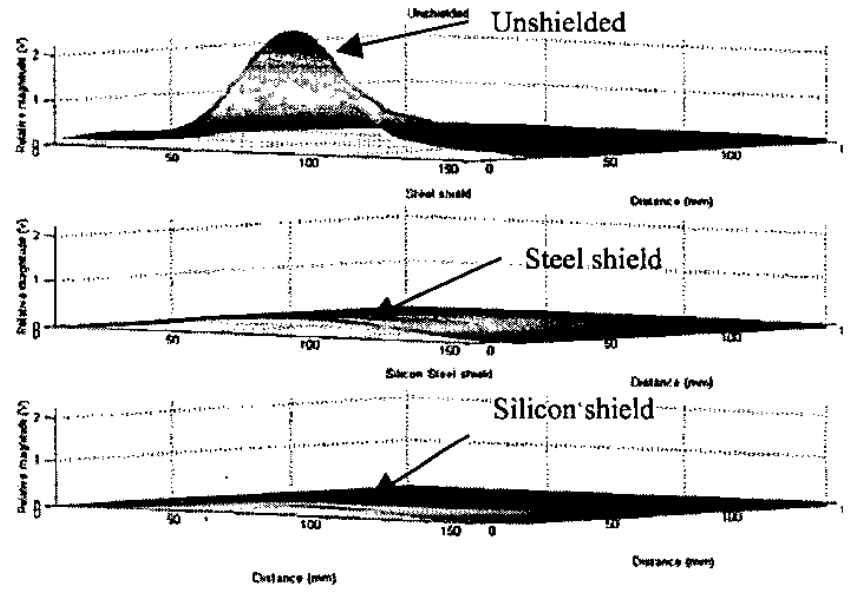

Fig.6. Shielded and unshielded plot comparisons.

The twin layer design has the advantage of further reduction of external interference, whilst additionally increasing the wanted signal output.

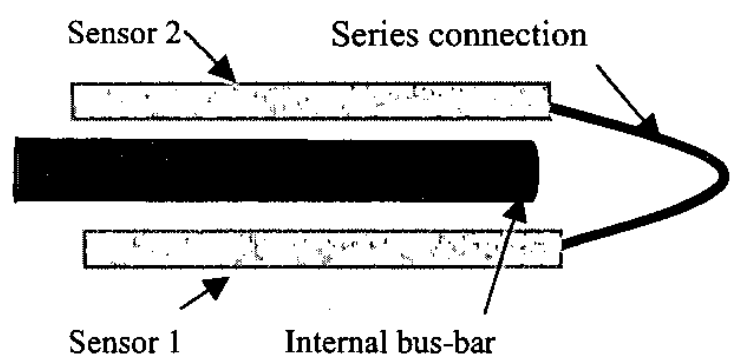

Fig.7. Twin layer configuration. 
Table 2 demonstrates a typical set of data utilizing $12 \times 45$ $\mathrm{mm}$ coils, concentrating on the comparisons between the single and the twin layer approach, with no shielding. Naturally the signal response is enhanced further when the internal shield is incorporated. These results indicate an increase in internal flux linkage and hence voltage pick up, with a further reduction in external interference, although, these values are subject to many variables within the system :

- Number of turns per coil.

- Individual coil separation.

- Sensor layer separation.

- Shield depth/dimensions.

- Outer case dimensions of the meter.

Table 2 (Comparisons in internal/external linkage between single/twin layer designs.)

\begin{tabular}{|c|c|c|c|}
\hline & $\begin{array}{l}\text { Internal } \\
(1.6 \mathrm{~mm})\end{array}$ & $\begin{array}{l}\text { External } \\
(20 \mathrm{~mm})\end{array}$ & $\begin{array}{r}\text { Percentage } \\
\text { difference (\%) }\end{array}$ \\
\hline $\begin{array}{l}\text { Single layer } \\
\text { (no shield) }\end{array}$ & $0.4 \mathrm{Vpk}-\mathrm{pk}$ & $0.06 \mathrm{Vpk}$-pk & $15 \%$ \\
\hline $\begin{array}{l}\text { Twin layer } \\
\text { (no shield) }\end{array}$ & 0.8Vpk-pk & $0.06 \mathrm{Vpk}-\mathrm{pk}$ & $7.5 \%$ \\
\hline
\end{tabular}

The additional flux linkage output is naturally established due to the extra sensor, the two sensors are able to work in conjunction with each other to further reject external interference, as the sensor layers essentially work as compensating coils.

As the sensors separation layer decreases, the internal flux linkage and hence the voltage output increases substantially, and of course this provides a reduction in external interference because the coils are moving further away from the external source within the current meter housed unit.

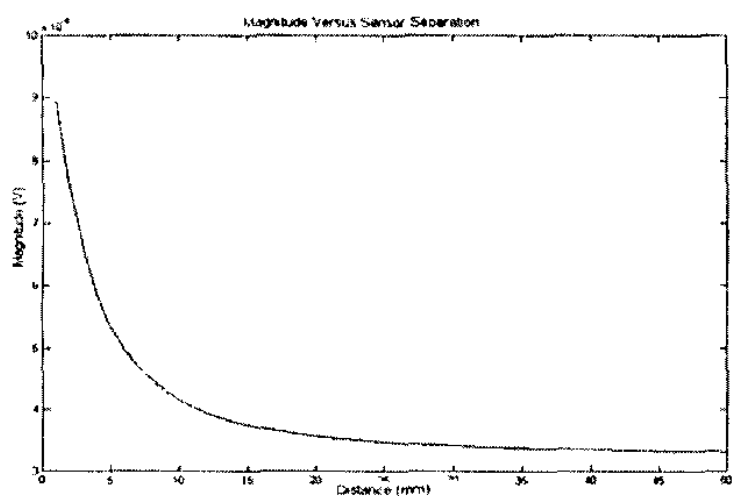

Fig.8. Magnitude variation as twin layer loop separation increases.

Figure 8 illustrates the response in terms of magnitude of the twin layer system as the layer separation is altered. It can be seen that the closer the layers become the larger the output magnitude. Although, there is a physical limitation to the loop separation, that of, the internal shield incorporated and the actual internal current carrying conductor. Naturally the twin layer design can be enhanced to multiple layers in an array formation either utilizing multiple boards, or a single multiple layered PCB.

The output is of course substantially larger than the single/twin layer design, unfortunately this design provides additional external interference due to the increase in sensitivity, although utilizing the $(79 \times 19 \mathrm{~mm})$ coils it is possible to achieve $200 \mathrm{mV}$ output signal with no additional amplification.

\section{V.RESULTS}

Table 3 demonstrates the results of a twin layer system incorporating each magnetic material in the internal shield configuration. The coils used have dimensions of $79 \times 19 \mathrm{~mm}$ per coil. Results indicate a considerable increase in voltage from utilizing the shields, this is because the flux from the internal conductor, tends to flow around the internal shield as a low reluctance path and couple further with the sensor.

Table 3 (Sensors output to internal/external linkage between single/twin layer designs with shielding, utilizing $72 \times 19 \mathrm{~mm}$ coils.)

\begin{tabular}{|c|c|c|c|}
\hline $\begin{array}{c}\text { Twin layer } \\
\text { design }\end{array}$ & $\begin{array}{c}\text { Internal } \\
(1.6 \mathrm{~mm})\end{array}$ & $\begin{array}{c}\text { External } \\
(20 \mathrm{~mm})\end{array}$ & $\begin{array}{c}\text { Percentage } \\
\text { difference }\end{array}$ \\
\hline No shield & $2.25 \mathrm{Vpk}-\mathrm{pk}$ & $\mathbf{0 . 2 1 0 V \mathrm { Vk } - \mathrm { pk }}$ & $9.33 \%$ \\
\hline Steel shield & $5.4 \mathrm{Vpk}$-pk & $\mathbf{0 . 0 7 6 V \mathrm { pk } - \mathrm { pk }}$ & $\mathbf{0 . 8} \%$ \\
\hline Silicon shield & $5.4 \mathrm{Vpk}-\mathrm{pk}$ & $\mathbf{0 . 0 7 6 V p k - p k}$ & $\mathbf{0 . 6} \%$ \\
\hline
\end{tabular}

It can be seen that by using each material it is possible to achieve the desired $<1 \%$ tolerance to external stray fields, which is required for domestic metering purposes. The results also demonstrate the similarities between the two magnetic shielding types, even though the material properties are quite different, the magnetic fields share similar responses.

Figures $9 \& 10$ were achieved by 3D simulation, hence the peaks seen in magnitude can not be realized in practice, due to the physical location of the sense coils. Figure 9 illustrates a side view of the 2D spatial response of a twin layer design. Each individual sensors peak response can be seen clearly in the boxed area. It can also be easily visualized at the labeled symmetrical point, how the magnitude varies with the layer separation. Figure 9 illustrates a layer separation of $18 \mathrm{~mm}$, providing a $6.3 \%$ value of full scale range, whilst figure 10 illustrates a layer separation of $6 \mathrm{~mm}$, which provides a $34 \%$ value of full scale range. 


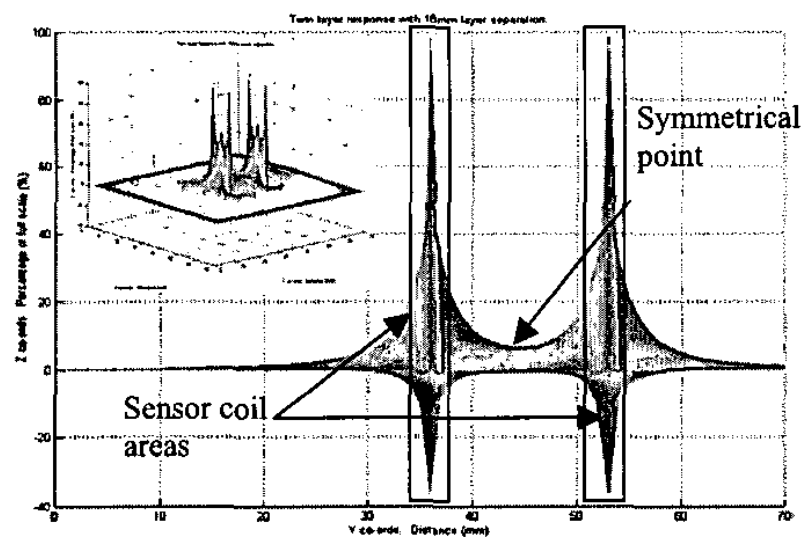

Fig.9. Twin layer configuration at $18 \mathrm{~mm}$ loop separation.

Although the twin layer design does provide an increase in the output signal with relation to the layer separation, there is a physical limitation to the separation, that of the internal conductor and sensor coils.

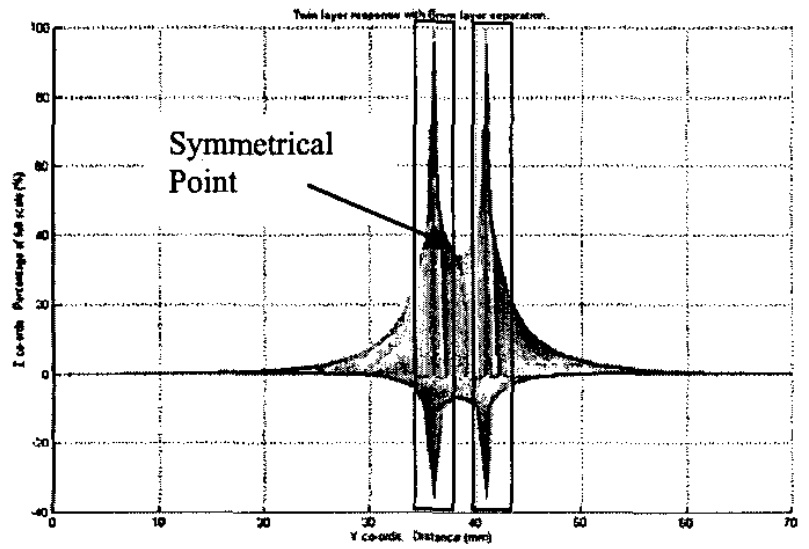

Fig.10. Twin layer configuration at $6 \mathrm{~mm}$ loop separation.

The sensor can measure both sinusoidal and nonsinusoidal A.C waveforms. Tests have also shown that the response of the device to input current is substantially linear over a wide dynamic range from approximately $(\approx 10 \mathrm{~mA}$ to $>$ 100A RMS), although the lower end signal response depends on the sensors characteristics and the noise level of the system. Figure 11 contains two plots, the top plot shows the sensors differentiated output response due to Faradays effect, and the bottom plot has two traces the first indicating the input current waveform from the shunt, and the second the sensors calibrated output response. It can be shown that once the sensors output has been signal conditioned, the output is virtually indistinguishable from the required shunt current input waveform.

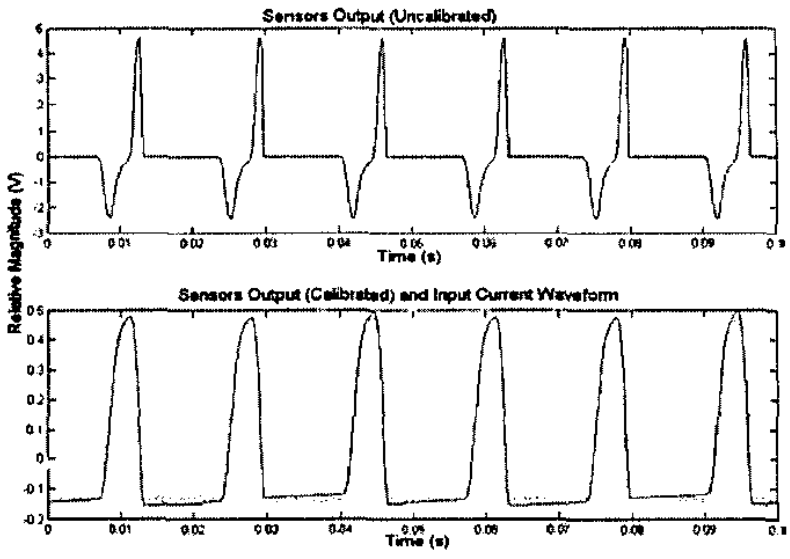

Fig.11. $50 \mathrm{~Hz}$ waveform, a half wave rectified signal.

Figure 12 demonstrates the sensors capabilities of measuring several waveforms, with various harmonic content. The figure illustrates a graph containing six different plots, each one containing two traces, the first being the actual input current shunt waveform and the second is the sensors response after calibration.
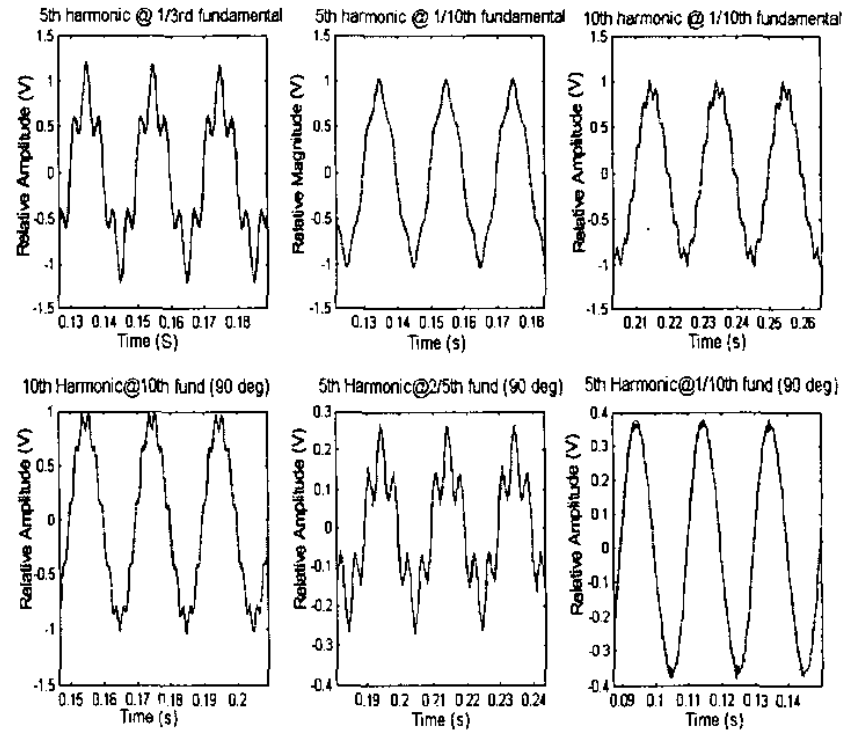

Fig.12. $50 \mathrm{~Hz}$ waveform, a half wave rectified signal.

The sensor demonstrates its ability to accurately reconstruct the original current waveform with virtually indistinguishable results. The waveforms consisted of a $50 \mathrm{~Hz}$ fundamental plus a variety of harmonic content up to and including the $20^{\text {th }}$ harmonic at an assortment of magnitudes and phase angles. 


\section{CONCLUSION}

This paper has introduced a current sensor design that can be implemented directly onto a PCB with the sensor picking up flux linkages in one plane of geometry only, whilst providing maximum noise immunity due to the coil configuration from external sources. The sensor offers a new design that has potential cost benefits over traditional approaches. Sensitivity plots have been examined in detail with variation in the peak response shown by varying the distribution of the coils. Two different shield configurations have been shown that can provide a sufficient degree of rejection to external sources. A possible twin layer coil design has also been discussed to improve further the performance of the system, in terms of increasing the internal signal whilst rejecting further possible external interference. Finally the sensor has shown the ability to accurately sense various waveforms of differing harmonic content.

\section{ACKNOWLEDGEMENTS}

The authors would like to thank Bob Mackin and Evelyn Shaw from Lancaster University for their contributions to this paper.

\section{REFERENCES}

[1] [1] Alternating current static watt-hour meters for active energy (class 1 and 2). European Standard EN 61036. October 1996

[2] [2] Improvements in or related to current measurement. UK Patent Application (GB 2353.598 A)

[3] [3] LJUBOMIR KOJOVIC, 'PCB Rogowski coils benefit relay protection'. IEEE computer applications in power. July 2002. VOL 15. ISSN : 0895-0156-02

[4] [4] LJUBOMIR KOJOVIC et al, 'United State Patent '. US $6,313,623$ B1. Nov $6^{\text {th }}, 2001$. 